\title{
Possible absence of trimeron correlations above the Verwey temperature in $\mathrm{Fe}_{3} \mathrm{O}_{4}$
}

\author{
H. Elnaggar $\odot,{ }^{1, *}$ R. Wang, ${ }^{1}$ S. Lafuerza, ${ }^{2}$ E. Paris, ${ }^{3}$ A. C. Komarek, ${ }^{4}$ H. Guo,${ }^{4}$ Y. Tseng, ${ }^{3}$ D. McNally, ${ }^{3}$ F. Frati, ${ }^{1}$ \\ M. W. Haverkort, ${ }^{5}$ M. Sikora, ${ }^{6}$ T. Schmitt, ${ }^{3}$ and F. M. F. de Groot ${ }^{1, \dagger}$ \\ ${ }^{1}$ Debye Institute for Nanomaterials Science, Utrecht University, Universiteitsweg 99, 3584 CA Utrecht, The Netherlands \\ ${ }^{2}$ European Synchrotron Radiation Facility, CS40220, F-38043 Grenoble Cedex 9, France \\ ${ }^{3}$ Photon Science Division, Paul Scherrer Institut, Forschungsstrasse 111, 5232 Villigen PSI, Switzerland \\ ${ }^{4}$ Max-Planck-Institute for Chemical Physics of Solids, Nöthnitzer Strasse 40, 01187 Dresden, Germany \\ ${ }^{5}$ Institut für Theoritiche Physik, Universität Heidelberg, Philosophenweg 19, 69120 Heidelberg, Germany \\ ${ }^{6}$ Academic Centre for Materials and Nanotechnology, AGH University of Science and Technology, Mickiewicza 30, 30-059 Krakow, Poland
}

(Received 5 February 2019; revised manuscript received 20 September 2019; accepted 17 January 2020; published 6 February 2020)

\begin{abstract}
The origin of the metal to insulator transition in $\mathrm{Fe}_{3} \mathrm{O}_{4}$ remains a challenge due to the complexity of the system: it is a mixed valent, strongly correlated system where many interactions such as Jahn-Teller distortion, exchange, and phonons are very close in energetics. A recent interpretation of the Verwey transition as an ordering of a three-site magnetic polaron, the trimeron, has been put forward. Here we investigate the existence of the trimeron correlations in the high-temperature phase of $\mathrm{Fe}_{3} \mathrm{O}_{4}$ using high-resolution iron $2 p 3 d$ resonant inelastic scattering magnetic linear dichroism. Guided by theoretical simulations, we reveal that the polarization dependence of the low-energy spin-orbital excitations is incompatible with tetragonal Jahn-Teller trimeron-type distortion. We conclude that the lowest-energy state of the high-temperature phase of $\mathrm{Fe}_{3} \mathrm{O}_{4}$ arises from an intricate interplay between trigonal crystal-field, exchange, and spin-orbit interactions.
\end{abstract}

DOI: 10.1103/PhysRevB.101.085107

\section{INTRODUCTION}

Magnetite $\left(\mathrm{Fe}_{3} \mathrm{O}_{4}\right)$ is one of the most abundant $\mathrm{Fe}$ bearing minerals on Earth. It is known to man as the first compass [1], an intriguing half-metallic oxide of interest to spintronics [2], and an archetype of Mott insulators with a switching time of few picoseconds [3,4]. Above the metal to insulator transition (the Verwey transition at $T_{V} \sim 125 \mathrm{~K}$ ), $\mathrm{Fe}_{3} \mathrm{O}_{4}$ has a cubic inverse spinel crystal structure $(F d \overline{3} m[5])$ containing two different $\mathrm{Fe}$ sites. $\mathrm{Fe}^{3+}$ ions reside in tetrahedral $\left(T_{d}\right)$ coordinated interstices (referred to as the A sites) while both $\mathrm{Fe}^{2+}$ and $\mathrm{Fe}^{3+}$ ions are in nearly octahedral $\left(O_{h}\right)$ coordinated interstices (referred to as the B sites). Verwey proposed that at $T_{V}$ an order to disorder transition takes place where the low-temperature ordering of the $\mathrm{B}$ site $\mathrm{Fe}^{3+}$ and $\mathrm{Fe}^{2+}$ ions melts, permitting relatively easy valency exchange by means of fast electron hopping [6]. Ever since this formulation, extensive efforts have been exerted to find evidence for the proposed charge ordering (and, later on, orbital ordering) and the accompanying distortion at the low-temperature phase [7-17].

A concrete result of the low-temperature phase crystal structure was obtained by a remarkable x-ray study on $\mathrm{Fe}_{3} \mathrm{O}_{4}$ microcrystals done by Senn et al. [18]. Based on the valence bond sum analysis of their diffraction results, charge ordering over a linear three-site Fe chain, termed the trimeron, was concluded. The trimeron-type correlations are associated

\footnotetext{
*h.m.e.a.elnaggar@uu.nl

†f.m.f.degroot@uu.nl
}

with compressive tetragonal Jahn-Teller distortion of the B site $\mathrm{FeO}_{6}$ octahedra. These distortions of the $\mathrm{Fe}^{2+}$ ions act parallel to the $a, b$, and $c$ axes of the high-temperature cubic lattice in a 5:5:6 ratio (see Fig. 1) that minimizes the strain in the highly connected spinel lattice. However, the expected entropy change for the transition from full disorder of $\mathrm{Fe}^{2+}$ and $\mathrm{Fe}^{3+}$ charges to the low-temperature trimeron structure is greater than the experimentally observed value (22.5 J K ${ }^{-1} \mathrm{~mol}^{-118}$ [18] versus $6.4 \mathrm{~J} \mathrm{~K}^{-1} \mathrm{~mol}^{-119}$ [19]). This large discrepancy suggests that the Fe B ions are not fully disordered in the high-temperature phase. The crucial question to follow is thus: are short-range trimeron correlations persistent at temperatures greater than $T_{V}$ ? It has remained a challenge to establish (or dismiss) the existence of the high-temperature trimeron correlations because of their dynamical nature and associated small local Jahn-Teller distortion which renders standard techniques such as x-ray diffraction, UV-Vis, and NMR spectroscopy ineffective.

In this paper, we present a high-resolution $\mathrm{Fe} 2 p 3 d$ resonant inelastic $x$ ray scattering magnetic linear dichroism (RIXS-MLD) study of $\mathrm{Fe}_{3} \mathrm{O}_{4}$ focusing on the hightemperature phase. The polarization and magnetic dependence of a low-energy excitation (centered at $\sim 200 \mathrm{meV}$ ) associated to the $\mathrm{Fe}^{2+} \mathrm{B}$ ions are revealed. Our extensive analysis of the polarization dependence uncovered the internal orbital character of the composite spin-orbital excitations. In contrast to previous propositions, we demonstrate that the local symmetry of the nominal $\mathrm{Fe}^{2+}$ ions above $T_{V}$ is trigonal. This casts some doubts regarding the interpretation of the Verwey transition as a long-range to a short-range order of the trimeron quasiparticle. 
(a)

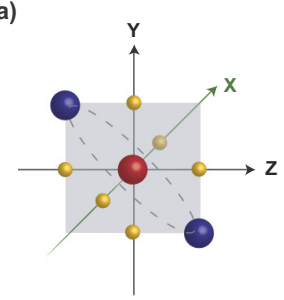

Tetragonal $\mathbf{X}$ (b)

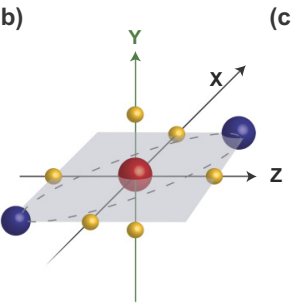

Tetragonal $\mathrm{Y}$ (c)

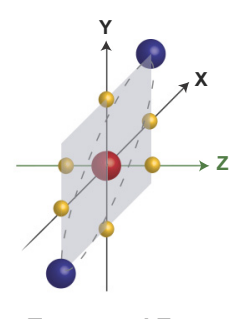

Tetragonal Z

$\mathrm{Fe}^{2+} \bigcirc \mathrm{Fe}^{3+} \bigcirc \mathrm{O}^{2-}$

FIG. 1. Tetragonal distortion associated with trimeron correlations as reported by Senn et al. [18]. The Jahn-Teller distortion at the $\mathrm{B}$ site $\mathrm{FeO}_{6}$ octahedra acts parallel to the $a$ axis [tetragonal $\mathrm{X}$ in panel (a)], $b$ axis [tetragonal $\mathrm{Y}$ in panel (b)], and $c$ axis [tetragonal $\mathrm{Z}$ in panel (c)] of the high-temperature cubic lattice in a 5:5:6 ratio.

\section{METHODS}

RIXS measurement at the $L$ edges of transition metals ( $2 p \rightarrow 3 d$ excitations) is a powerful tool sensitive to charge, orbital, spin, and lattice degrees of freedom [20-22]. The chemical selectivity provided on resonance enables RIXS to probe independently the electronic properties stemming from different sites in a mixed valent system. By appropriate tuning of the incident energy to the lowest-energy peak of $L_{3}$, we can gain sensitivity to the nominal $\mathrm{Fe}^{2+}$ ions in $\mathrm{Fe}_{3} \mathrm{O}_{4}$ [23-30]. Low-energy spin-orbital excitations are revealed in the energy-loss slices. They provide a direct probe of small static and dynamical crystal-field distortions at the $\mathrm{Fe}^{2+}$ ions, i.e., of polarons. Despite the impressive progress in the experimental resolution of RIXS beamlines, it remains a challenge to resolve close-lying spin-orbital states, which sheds doubts on the spectral interpretation. The discrimination power gained by coupling RIXS measurements with magnetic dichroism can provide a distinct solution to the ground state.

Fe $L_{3}$-edge $x$-ray absorption spectroscopy (XAS) and RIXS measurements were carried out at the ADRESS beamline of the Swiss Light Source at the Paul Scherrer Institut, Switzerland [31]. All measurements were performed at $170 \mathrm{~K}$ in a normal incidence geometry, i.e., with the incoming beam impinging at an angle of $90^{\circ}$ with respect to the sample surface. The scattering angle was set to $2 \theta=130^{\circ}$. The combined energy resolution was $\sim 76.2 \mathrm{meV}$ determined by the full width at half maximum of the elastic scattering peak from a carbon tape reference. The radiation source is a fixed-gap Apple-II type undulator [32], producing left and right circular polarized light as well as linear polarized light. A permanent gold coated $\mathrm{NdFeB}$ magnet with a magnetic flux density on the surface of $0.4 \mathrm{~T}$ was used to saturate the magnetization (see Supplemental Material [33]).

\section{RESULTS AND DISCUSSION}

High-resolution $\mathrm{Fe} 2 p 3 d$ RIXS measurements are presented in Fig. 2. The $L_{3}$ XAS spectrum and RIXS energy-loss slice at the first peak of $L_{3}$ are shown to the right and bottom of the RIXS map, respectively. At an incidence energy of $706.1 \mathrm{eV}$ (labeled $E_{I}$ ), the resonance is dominantly selective

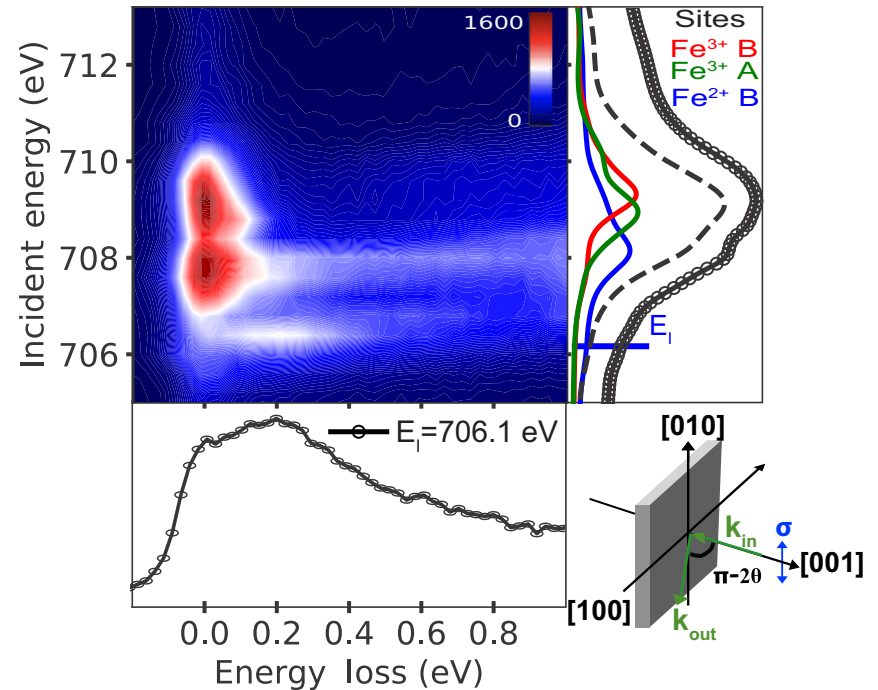

FIG. 2. Fe $L_{3}$ RIXS map of $\mathrm{Fe}_{3} \mathrm{O}_{4}$. The $L_{3}$ XAS signal is shown on the right. Theoretical contributions of the three $\mathrm{Fe}$ ions (solid lines) and the stoichiometric summation of the three Fe ions (dashed line) are plotted. A broad peak can be observed at $\boldsymbol{E}_{\boldsymbol{I}}=706.1 \mathrm{eV}$. This corresponds to the first peak of the $L_{3}$ XAS signal labeled $E_{I}$. The RIXS cut at $\boldsymbol{E}_{\boldsymbol{I}}$ is shown in the bottom panel. A sketch of the scattering geometry is shown in the right bottom corner where $\sigma$ is the vertical incident polarization, $\boldsymbol{k}_{\text {in }}$ and $\boldsymbol{k}_{\text {out }}$ are the incident and scattered wave vectors, and $2 \theta$ is set to $130^{\circ}$.

to the nominal $\mathrm{Fe}^{2+}$ ions as concluded by the fitting of XAS and X-ray magnetic circular dichroism (XMCD) spectra $[23,24,26,30,33,40]$. The corresponding RIXS spectrum exhibits a broad low-energy peak centered at $200 \mathrm{meV}$. In order to interpret this broad feature, an examination of the $\mathrm{Fe}^{2+}$ ground state is required.

The ${ }^{5} D_{4}$ atomic term symbol of an $\mathrm{Fe}^{2+}$ ion forms the ${ }^{5} T_{2 g}$ and ${ }^{5} E_{g}$ terms in a cubic symmetry with ${ }^{5} T_{2 g}$ being the ground state for an $O_{h}$ crystal field. The ${ }^{5} T_{2 g}$ states are further split and mixed by smaller perturbations such as Jahn-Teller distortion, exchange, and spin-orbit interaction. The broad peak is therefore composed mainly of excitations within the 15 -fold ${ }^{5} T_{2}$ microstates [41]. The details of the ground-state determination are presented in the Supplemental Material [33]. Theoretical prediction of the energy positions of these states is shown in Fig. 3(b) for a Jahn-Teller tetragonally distorted $\mathrm{Fe}^{2+}$ ion along the [001] direction (red) and for a trigonally distorted $\mathrm{Fe}^{2+}$ ion along the [111] direction (green), respectively. Only minor differences in the energy positions between the two types of distortions are predicted. These differences are undetectable with current state-of-the-art experimental resolution. We show in the following that RIXS dichroism experiments provide a unique approach to overcome the uncertainty in energy positions by providing strong constraints based on symmetry.

We initially measured the RIXS-MLD signal at $\boldsymbol{E}_{\boldsymbol{I}}=$ $706.1 \mathrm{eV}$ with the external magnetic field $(\boldsymbol{B})$ aligned to the incident wave vector, i.e., $\boldsymbol{B}\left\|\boldsymbol{k}_{\text {in }}\right\|$ [001] as shown in Fig. 3(a). The dichroism signal was recorded by rotating the incident polarization $\left(\boldsymbol{\epsilon}_{\text {in }}\right)$ from linear vertical (referred to as $\phi=0^{\circ}$ ) to linear horizontal (referred to as $\phi=90^{\circ}$ ). 

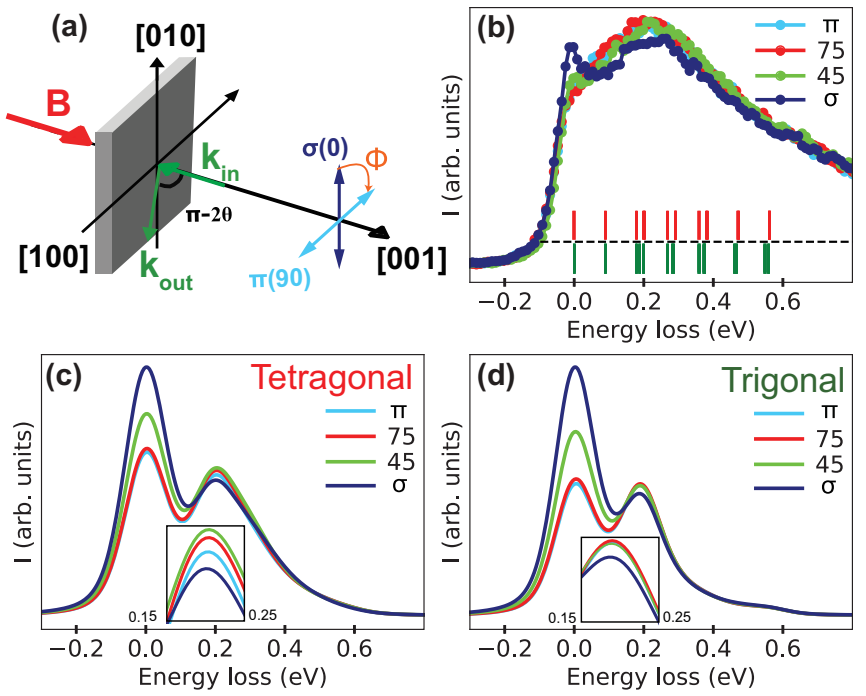

FIG. 3. Fe $2 p 3 d$ RIXS-MLD at $\boldsymbol{E}_{\boldsymbol{I}}=706.1 \mathrm{eV}$ results for $\boldsymbol{B} \|$ [001] orientation. (a) Sketch of the experimental configuration. $\boldsymbol{k}_{\text {in }}$ and $\boldsymbol{k}_{\text {out }}$ are the incident and scatter wave vectors, respectively. $\boldsymbol{\sigma}$ and $\boldsymbol{\pi}$ are the vertical and horizontal incident polarizations. $\boldsymbol{B}$ is the external magnetic field. (b) Experimental RIXS angular dependence measured as a function of the angle $(\phi)$ between the incident polarization and the vertical direction. Calculations of the $\mathrm{Fe} 2 p 3 d$ RIXS angular dependence in $\mathrm{Fe}_{3} \mathrm{O}_{4}$ with the nominal $\mathrm{Fe}^{2+}$ ions in (c) tetragonal symmetry and (d) trigonal symmetry.

The dichroism signal is composed of contributions from both structural and magnetic effects. This is in fact a feature of any dichroic measurement recorded as $I_{\text {Dichroism }}=$ $I\left[\boldsymbol{B}\left(90^{\circ}\right), \boldsymbol{\epsilon}_{\text {in }}\left(\phi=0^{\circ}\right)\right]-I\left[\boldsymbol{B}\left(90^{\circ}\right), \boldsymbol{\epsilon}_{\text {in }}(\phi)\right]$. We note that the structural and magnetic dichroism signals are intrinsically coupled for $\mathrm{Fe}_{3} \mathrm{O}_{4}$ because its ground state is naturally magnetic. A regular RIXS-LD measurement would result in an average of the RIXS-MLD signal over the magnetic domains ( $\langle 111\rangle$ directions) probed by the $4 \times 55-\mu \mathrm{m} \mathrm{x}$-ray beam.

A small angular dependence is observed in this configuration as shown in Fig. 3(b). Here the angle between $\boldsymbol{B}$ and $\epsilon_{\text {in }}$ does not change as a function of the $\phi$ rotation, which minimizes the magnetic contribution to the dichroism signal. In addition, the deviation of the $\mathrm{Fe}^{2+}$ local site symmetry from $O_{h}$ is small, implying that the structural dichroism is also expected to be small. To the contrary, a stronger RIXS-MLD signal is observed when $\boldsymbol{B}$ is aligned parallel to $\left[-\frac{1}{2} \frac{\sqrt{3}}{2} 0\right]$ (i.e., displacing the external magnetic field $30^{\circ}$ from the highsymmetry [010] direction) as shown in Fig. 4(b). Now the angle between $\boldsymbol{B}$ and $\boldsymbol{\epsilon}_{\text {in }}$ changes as a function of $\phi$, leading to a significant magnetic dichroism contribution. As a matter of fact, we chose to orient $\boldsymbol{B}$ in such a low-symmetry direction to maximize site anisotropy effects and hence the dichroism signal.

In order to uniquely identify the ground-state symmetry of the $\mathrm{Fe}^{2+}$ ions, the RIXS-MLD measurements were compared to theoretical simulations. We used the quantum many-body program QUANTY [42-44] to simulate the $2 p 3 d$ RIXS spectra of $\mathrm{Fe}_{3} \mathrm{O}_{4}$. The Hamiltonian used for the calculations consists of the following terms: (i) Coulomb interaction, (ii) crystalfield potential, (iii) spin-orbit coupling, and (iv) magnetic (a)
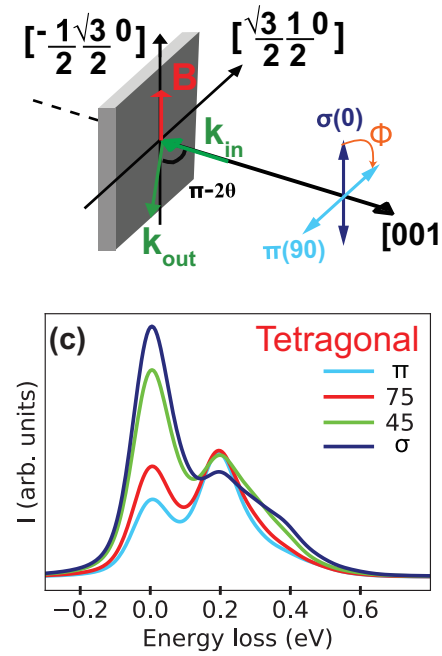
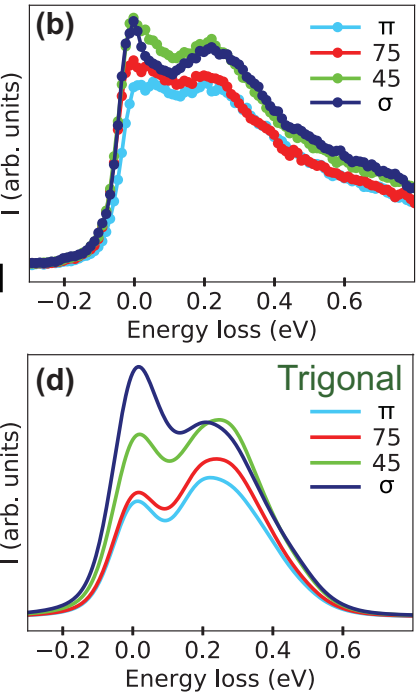

FIG. 4. Fe $2 p 3 d$ RIXS-MLD at $\boldsymbol{E}_{\boldsymbol{I}}=706.1 \mathrm{eV}$ results for $\boldsymbol{B} \|$ $\left[-\frac{1}{2} \frac{\sqrt{3}}{2} 0\right]$ orientation. (a) Sketch of the experimental configuration. $\boldsymbol{k}_{\text {in }}$ and $\boldsymbol{k}_{\text {out }}$ are the incident and scatter wave vectors, respectively. $\boldsymbol{\sigma}$ and $\boldsymbol{\pi}$ are the vertical and horizontal incident polarizations. $\boldsymbol{B}$ is the external magnetic field. (b) Experimental RIXS angular dependence measured as a function of the angle $(\phi)$ between the incident polarization and the vertical direction. Calculations of the $\mathrm{Fe} 2 p 3 d$ RIXS angular dependence in $\mathrm{Fe}_{3} \mathrm{O}_{4}$ with the nominal $\mathrm{Fe}^{2+}$ ions in (c) tetragonal symmetry and (d) trigonal symmetry.

exchange interaction. The $d-d(p-d)$ multipole part of the Coulomb interaction was scaled to $70 \%(80 \%)$ of the Hartree-Fock values of the Slater integral. The parameters used for the calculations are reported in Tables S1, S2, and S3 of the Supplemental Material [33].

The low-energy feature can be best interpreted as a $d d$ excitation in the presence of a small Jahn-Teller distortion. In a pure octahedral coordination, the weak spin-orbit $(66.5 \mathrm{meV})$ and exchange $(90 \mathrm{meV})$ interactions cannot split the 15 -fold ${ }^{5} T_{2 g}$ multiplet enough to produce a pronounced peak centered at $200 \mathrm{meV}$ (refer to Fig. S14 of the Supplemental Material [33]). We emphasize that such weak distortions do not significantly modify the XAS and XMCD spectral shapes and hence they could only be effectively studied with RIXS-MLD measurements (see Sec. IV of the Supplemental Material [33]).

A widely appealing proposition for such a symmetry lowering at the $\mathrm{Fe}^{2+}$ sites is the presence of trimeron correlations inherited from the low-temperature phase that induce dynamical local tetragonal distortion above $T_{V}$. Figures 3(c) and 4(c) show the calculated RIXS angular dependence predicted for this trimeron-type distortion in the scattering geometry with $\boldsymbol{B}$ aligned $\|$ [001] [Fig. 3(c)] and that with $\boldsymbol{B}$ aligned $\|\left[-\frac{1}{2} \frac{\sqrt{3}}{2} 0\right]$ [Fig. 4(c)]. We note that the horizontal polarization results agree reasonably with the experiment in both magnetic configurations; however, the full angular evolution is inconsistent with the trimeron scenario. This partial agreement explains the assignment of the 200-meV feature to tetragonal distortion in recent work based on the result of the horizontal polarization RIXS measurement [17] and highlights the importance of a systematic magnetic angular dependence measurement. 
Another possibility for the symmetry lowering is the presence of a trigonal Jahn-Teller distortion at the $\mathrm{Fe}^{2+}$ sites along the $\langle 111\rangle$ axes of the high-temperature cubic phase. Simulations using trigonal distortion capture the angular evolution of the RIXS cross section well as illustrated in Figs. 3(d) and 4(d). A minor discrepancy between the calculated and experimental elastic line intensity can be seen systematically. We attribute this discrepancy to self-absorption effects. Selfabsorption of the emitted $\mathrm{x}$ rays dominates at zero energy loss in particular when the incident energy is tuned to the lowest-energy peak of the $L_{3}$ edge. This leads to the decrease of the experimental elastic peak intensity with respect to the simulations. We remark that the self-absorption effect is different between the measurements presented in Figs. 3 and 4, which makes a direct comparison between the intensity of the elastic peaks in the two cases difficult. The orientation of the magnetic field modifies the absorption cross section and hence the reabsorption of the emitted photons is modified as well. We have therefore focused our analysis on the inelastic 200$\mathrm{meV}$ peak that is significantly less affected by self-absorption.

\section{CONCLUSION}

In view of the diverging interpretations of the Verwey transition [45,46], we address hereafter the implications of our finding. Combining RIXS data and theoretical modeling, we unveiled how the angular dependence of spin-orbital entangled excitations at the $\mathrm{Fe}^{2+}$ ions establishes the presence of local trigonal Jahn-Teller distortion. Hence our results suggest the absence of the trimeron quasiparticles above $T_{V}$. The trigonal distortion is consistent with the point-group symmetry of the $\mathrm{Fe} \mathrm{B}$ sites and the magnitude of the distortion we find $\left[D_{\sigma}=67(10) \mathrm{meV}\right]$ is in agreement with the static crystal-field splitting from DFT calculations. However, as the distortion magnitude lies close to the phonon energies of $\mathrm{Fe}_{3} \mathrm{O}_{4}$ [47-51], the trigonal distortion is presumably coupled to the lattice vibrations, leading to short-range trigonal based correlations. We foresee that performing RIXS-MLD measurements across the phase transition on detwinned microcrystals [18] or thin films [52] in search for the intricate local symmetry transformation from trigonal to tetragonal distortion at the Verwey transition would provide deep insights into the order parameter of the Verwey transition in $\mathrm{Fe}_{3} \mathrm{O}_{4}$.

\section{ACKNOWLEDGMENTS}

We thank A. Bosak and M. Hussein for their help with the $\mathrm{x}$-ray-diffraction measurements and sample characterization. A.v.d.E. and S.D. are thanked for their help with designing the sample holder for the synchrotron measurements. We are grateful for the fruitful discussions with A. Juhin, Ch. Brouder, M.-A. Arrio, Ph. Sainctavit, H. Y. Huang, D. J. Huang, and H. Tjeng. We acknowledge the support of the Laue laboratory at the Paul Scherrer Institut (PSI). We thank Thomas Schmidt for his valuable assistance with the experiment. The synchrotron experiments have been performed at the Advanced Resonant Spectroscopies (ADRESS) beamline of the Swiss Light Source at the PSI. The work at PSI is supported by the Swiss National Science Foundation through the National Centres of Competence in Research (NCCR) Materials' Revolution (MARVEL) and the Sinergia network Mott Physics Beyond the Heisenberg Model. M.S. acknowledges support from National Science Center of Poland (Grant No. 2014/14/E/ST3/00026). The research in Dresden is supported by the Deutsche Forschungsgemeinschaft through Grant No. 320571839. This work was financed by the European Research Council XRAYonACTIVE advanced Grant No. 340279.
[1] W. Lowrie, Fundamentals of Geophysics, 2nd ed. (Cambridge University, Cambridge, England, 2007).

[2] J. P. Hong, S. B. Lee, Y. W. Jung, J. H. Lee, K. S. Yoon, K. W. Kim, C. O. Kim, C. H. Lee, and M. H. Jung, Appl. Phys. Lett. 83, 1590 (2003).

[3] N. Pontius, T. Kachel, C. Schüßler-Langeheine, W. F. Schlotter, M. Beye, F. Sorgenfrei, C. F. Chang, A. Föhlisch, W. Wurth, P. Metcalf et al., Appl. Phys. Lett. 98, 182504 (2011).

[4] S. de Jong, R. Kukreja, C. Trabant, N. Pontius, C. F. Chang, T. Kachel, M. Beye, F. Sorgenfrei, C. H. Back, B. Bräuer et al., Nat. Mater. 12, 882 (2013).

[5] C. Haavik, S. Stolen, H. Fjellvag, M. Hanfland, and D. Hausermann, Am. Mineral. 85, 514 (2000).

[6] E. J. W. Verwey, Nature (London) 144, 327328 (1939).

[7] W. C. Hamilton, Phys. Rev. 110, 1050 (1958).

[8] T. Yamada, K. Suzuki, and S. Chikazumi, Appl. Phys. Lett. 13, 172 (1968).

[9] R. Hargrove and W. Kundig, Solid State Commun. 8, 303 (1970).

[10] L. V. Gasparov, D. B. Tanner, D. B. Romero, H. Berger, G. Margaritondo, and L. Forró, Phys. Rev. B 62, 7939 (2000).

[11] P. Novák, H. Štěpánková, J. Englich, J. Kohout, and V. A. M. Brabers, Phys. Rev. B 61, 1256 (2000).
[12] J. García, G. Subías, M. G. Proietti, J. Blasco, H. Renevier, J. L. Hodeau, and Y. Joly, Phys. Rev. B 63, 054110 (2001).

[13] G. Subías, J. García, J. Blasco, M. Grazia Proietti, H. Renevier, and M. Concepción Sánchez, Phys. Rev. Lett. 93, 156408 (2004).

[14] M. S. Senn, J. P. Wright, and J. P. Attfield, J. Korean Phys. Soc. 62, 1372 (2013).

[15] A. Bosak, D. Chernyshov, M. Hoesch, P. Piekarz, M. Le Tacon, M. Krisch, A. Kozłowski, A. M. Oleś, and K. Parlinski, Physical Review X 4, 011040 (2014).

[16] M. Taguchi, A. Chainani, S. Ueda, M. Matsunami, Y. Ishida, R. Eguchi, S. Tsuda, Y. Takata, M. Yabashi, K. Tamasaku et al., Phys. Rev. Lett. 115, 256405 (2015).

[17] H. Y. Huang, Z. Y. Chen, R. P. Wang, F. M. De Groot, W. B. Wu, J. Okamoto, A. Chainani, J. S. Zhou, H. T. Jeng, G. Y. Guo et al., Nat. Commun. 8, 15929 (2017).

[18] M. S. Senn, J. P. Wright, and J. P. Attfield, Nature (London) 481, 173 (2012).

[19] Z. Tarnawski, A. Wiecheć, M. Madej, D. Nowak, D. Owoc, G. Krol, Z. Kakol, L. Kolwiczchodak, A. Kozlowski, and T. Dawid, Acta Phys. Pol. A 106, 771 (2004).

[20] L. J. P. Ament, M. van Veenendaal, T. P. Devereaux, J. P. Hill, and J. van den Brink, Rev. Mod. Phys. 83, 705 (2011). 
[21] V. Bisogni, S. Catalano, R. J. Green, M. Gibert, R. Scherwitzl, Y. Huang, V. N. Strocov, P. Zubko, S. Balandeh, J. M. Triscone et al., Nat. Commun. 7, 13017 (2016).

[22] Y. Lu, D. Betto, K. Fürsich, H. Suzuki, H.-H. Kim, G. Cristiani, G. Logvenov, N. B. Brookes, E. Benckiser, M. W. Haverkort et al., Physical Review X 8, 031014 (2018).

[23] P. Kuiper, B. Searle, L.-C. Duda, R. Wolf, and P. van der Zaag, J. Electron. Spectrosc. Relat. Phenom. 86, 107 (1997).

[24] R. A. D. Pattrick, G. van der Laan, C. M. B. Henderson, P. Kuiper, E. Dudzik, and D. J. Vaughan, Eur. J. Mineral. 14, 1095 (2002).

[25] E. Goering, S. Gold, M. Lafkioti, and G. Schütz, Europhys. Lett. 73, 97 (2006).

[26] E. Arenholz, G. van der Laan, R. V. Chopdekar, and Y. Suzuki, Phys. Rev. B 74, 094407 (2006).

[27] E. Goering, M. Lafkioti, S. Gold, and G. Schütz, J. Magn. Magn. Mater. 310, e249 (2007).

[28] C. Carvallo, P. Sainctavit, M. A. Arrio, N. Menguy, Y. Wang, G. Ona-Nguema, and S. Brice-Profeta, Am. Mineral. 93, 880 (2008).

[29] G. van der Laan and E. Arenholz, Eur. Phys. J.: Spec. Top. 169, 187 (2009).

[30] B. Liu, C. Piamonteze, M. U. Delgado-Jaime, R.-P. Wang, J. Heidler, J. Dreiser, R. Chopdekar, F. Nolting, and F. M. F. de Groot, Phys. Rev. B 96, 054446 (2017).

[31] V. N. Strocov, T. Schmitt, U. Flechsig, T. Schmidt, A. Imhof, Q. Chen, J. Raabe, R. Betemps, D. Zimoch, J. Krempasky et al., J. Synchrotron Radiat. 17, 631 (2010).

[32] S. Sasaki, K. Kakuno, T. Takada, T. Shimada, K. ichi Yanagida, and Y. Miyahara, Nucl. Instrum. Methods Phys. Res. 331, 763 (1993).

[33] See Supplemental Material at http://link.aps.org/supplemental/ 10.1103/PhysRevB.101.085107 for details about the simulations, which includes Refs. [34-39].

[34] C. J. Ballhausen, Introduction to Ligand Field Theory, McGrawHill Series in Advanced Chemistry (McGraw-Hill, New York, 1962).
[35] P. H. Butler, Point Group Symmetry Applications, 1st ed. (Springer, New York, 1981), p. 576.

[36] P. Carra, B. T. Thole, M. Altarelli, and X. Wang, Phys. Rev. Lett. 70, 694 (1993).

[37] F. de Groot and A. Kotani, Core Level Spectroscopy of Solids, 1st ed. (CRC, Boca Raton, FL, 2008).

[38] S. S. Gupta, H. Wadati, and G. A. Sawatzky, Europhys. Lett. 93, 47008 (2011).

[39] A. Juhin, C. Brouder, and F. M. F. de Groot, Open Phys. 12, 323 (2014).

[40] V. N. Antonov, B. N. Harmon, and A. N. Yaresko, Phys. Rev. B 67, 024417 (2003).

[41] Distortion and spin-orbit coupling can mix some of the ${ }^{5} E_{g}$ character within the ${ }^{5} T_{2 g}$.

[42] M. W. Haverkort, M. Zwierzycki, and O. K. Andersen, Phys. Rev. B 85, 165113 (2012).

[43] Y. Lu, M. Höppner, O. Gunnarsson, and M. W. Haverkort, Phys. Rev. B 90, 085102 (2014).

[44] M. W. Haverkort, G. Sangiovanni, P. Hansmann, A. Toschi, Y. Lu, and S. Macke, Europhys. Lett. 108, 57004 (2014).

[45] F. Walz, J. Phys.: Condens. Matter 14, R285 (2002).

[46] J. García and G. Subías, J. Phys.: Condens. Matter 16, R145 (2004).

[47] P. Piekarz, K. Parlinski, and A. M. Oleś, Phys. Rev. Lett. 97, 156402 (2006).

[48] P. Piekarz, K. Parlinski, and A. M. Oleś, Phys. Rev. B 76, 165124 (2007).

[49] S. Borroni, G. S. Tucker, F. Pennacchio, J. Rajeswari, U. Stuhr, A. Pisoni, J. Lorenzana, H. M. Rønnow, and F. Carbone, New J. Phys. 19, 103013 (2017).

[50] J. Cumby and J. P. Attfield, Nat. Commun. 8, 14235 (2017).

[51] T. Haupricht, R. Sutarto, M. W. Haverkort, H. Ott, A. Tanaka, H. H. Hsieh, H. J. Lin, C. T. Chen, Z. Hu, and L. H. Tjeng, Phys. Rev. B 82, 035120 (2010).

[52] A. Tanaka, C. F. Chang, M. Buchholz, C. Trabant, E. Schierle, J. Schlappa, D. Schmitz, H. Ott, P. Metcalf, L. H. Tjeng et al., Phys. Rev. Lett. 108, 227203 (2012). 